\title{
Performance of SKA as an air shower observatory
}

S. Buitink, ${ }^{1,2, *}$ A. Corstanje, ${ }^{1,2}$ H. Falcke, ${ }^{2,3,4}$ B. M. Hare, ${ }^{5}$ J. R. Hörandel, ${ }^{1,2,3}$

T. Huege, ${ }^{1,6}$ C. W. James, ${ }^{7}$ G. K. Krampah, ${ }^{1}$ K. Mulrey, ${ }^{1}$ P. Mitra, ${ }^{1}$ A. Nelles, ${ }^{8,9}$

H. Pandya, ${ }^{1}$ J. P. Rachen, ${ }^{1}$ O. Scholten, ${ }^{10}$ S. ter Veen, ${ }^{2,4}$ S. Thoudam, ${ }^{11}$

T. N. G. Trinh ${ }^{12}$ and T. Winchen ${ }^{13}$

${ }^{1}$ Astrophysical Institute, Vrije Universiteit Brussel, Pleinlaan 2, 1050 Brussels, Belgium

${ }^{2}$ Department of Astrophysics/IMAPP, Radboud University, P.O. Box 9010, 6500 GL Nijmegen, The Netherlands

${ }^{3}$ Nikhef, Science Park 105, 1098 XG Amsterdam, The Netherlands

${ }^{4}$ Netherlands Institute of Radio Astronomy (ASTRON), Postbus 2, 7990 AA Dwingeloo, The Netherlands

${ }^{5}$ University of Groningen, Kapteyn Astronomical Institute, Groningen, 9747 AD, Netherlands

${ }^{6}$ Institute for Astroparticle Physics, Karlsruhe Institute of Technology (KIT), P.O. Box 3640, 76021, Karlsruhe, Germany

${ }^{7}$ Curtin University, International Centre for Radio Astronomy Research, Bentley, WA 6102, Australia

${ }^{8}$ DESY, Platanenallee 6, 15738 Zeuthen, Germany

${ }^{9}$ ECAP, Friedrich-Alexander-University Erlangen-Nürnberg, 91058 Erlangen, Germany

${ }^{10}$ Interuniversity Institute for High-Energy, Vrije Universiteit Brussel, Pleinlaan 2, 1050 Brussels, Belgium

${ }^{11}$ Department of Physics, Khalifa University, PO Box 127788, Abu Dhabi, United Arab Emirates

${ }^{12}$ Department of Physics, School of Education, Can Tho University Campus II, 3/2 Street, Ninh Kieu District, Can Tho City, Vietnam

${ }^{13}$ Max-Planck-Institut für Radioastronomie, Auf dem Hügel 69, 53121 Bonn

E-mail: stijn.buitink@vub.be

The low frequency segment of SKA in Australia will have an extremely dense antenna array spanning an area of roughly $0.5 \mathrm{~km}^{2}$. It offers unique possibilities for high-resolution observations of air showers. Compared to LOFAR, it will have a much more homogeneous ground coverage, an increased frequency bandwidth (50-350 MHz), and the possibility to continuously observe with nearly $100 \%$ duty cycle. SKA will observe air showers in the range $10^{16} \mathrm{eV}-10^{18} \mathrm{eV}$ with a reconstruction resolution on $X_{\max }$ of around $10 \mathrm{~g} / \mathrm{cm}^{2}$. This allows for a high-precision study of mass composition in the energy regime where a transition is expected from Galactic to extragalactic origin. In addition, SKA will be able to put constraints on hadronic interaction models, which is crucial for interpreting the data in this complex energy range. In this talk, we will show the results of a full detector simulation and demonstrate the capabilities of SKA, including energy and $X_{\max }$ reconstruction, as well as more advanced methods to constrain the shape of the longitudinal development of air showers.

$3^{\text {th }}$ International Cosmic Ray Conference (ICRC 2021)

July 12 th - 23rd, 2021

Online - Berlin, Germany

*Presenter 


\section{The Square Kilometer Array}

On 29 June 2021 the start of the construction of the Square Kilometer Array (SKA) was officially approved. The SKA will consist of two telescope arrays: a mid-frequency site in South Africa, and a low-frequency site in Australia. It will support a broad range of science, including cosmology, the high-energy universe, magnetism, pulsars, and the search for extraterrestrial life [1].

The compact core of SKA-low, where 57,344 antennas will be constructed in a circular area with a diameter of $1 \mathrm{~km}$ offers excellent conditions for the radio detection of extended air showers [2]. The site is located $700 \mathrm{~km}$ north of Perth in a remote area with a very low level of anthropogenic radio frequency interference. The array will consist of dual-polarised log-periodic antennas that operate in the $50-350 \mathrm{MHz}$ range.

The extremely densely populated core sets SKA apart from other cosmic-ray radio observatories, which instead focus on maximizing the antenna spacing to cover a large area, required to reach the highest energies [3, 4]. Like LOFAR, the strength of SKA lies in performing high-resolution observations at energies below the ankle [5, 6]. However, SKA can surpass LOFAR on many fronts.

The core of SKA is slightly larger than that of LOFAR, but more importantly it has a much more homogeneous antenna coverage. The antennas have a larger bandwidth reaching up to 350 $\mathrm{MHz}$, compared to $80 \mathrm{MHz}$ for LOFAR. The combination of using shorter wavelength with more antennas naturally leads to a higher resolution reconstruction of the shower properties.

Figure 1 demonstrates the dramatic difference between LOFAR and SKA. At LOFAR, showers are observed with hundreds of antennas simultaneously. However, because they are arranged in circular stations, the coverage is inhomogeneous. The shape of the radiation pattern on the ground can be obtained by fitting the data to simulations [7]. At the SKA, on the other hand, the full pattern will appear directly. This will allow the most precise test imaginable to current state-of-the-art radio simulation codes like CoREAS [8] and ZHAireS [9].

Unlike LOFAR, SKA will be able to perform shower observation continuously with a duty cycle of near $100 \%$, stretching the energy range to higher values. At the same time, the homogeneous and dense layout allows for interferometric techniques that make it possible to have good detection efficiency down to much lower energies [10,11]. The SKA will observe from the ankle down to at least $10^{16} \mathrm{eV}$, and possibly even close to the knee.

Another advantage is that the SKA is in the southern hemisphere. Although large-scale anisotropies at sub-ankle energies are not generally expected, there currently exists tension between mass composition results of the Pierre Auger observatory in the south, and the Telescope Array and LOFAR in the north [6]. A combined analysis of LOFAR and SKA data will determine if the discrepancy is of astrophysical origin or due to currently unknown systematic uncertainties.

The vast amount of data - SKA observes individual showers with thousands of antennas - can be used to reconstruct not only the shower energy and $X_{\max }$ but also the shape of the longitudinal evolution of the shower. This has importance both for the analysis of mass composition of cosmic rays and to constrain the uncertainties in the hadronic physics governing the shower evolution. While the current generation of simulation codes is simply not fast enough to study all these possibilities in detail, we demonstrate that radio observations can indeed be used to measure the shape of longitudinal evolution of the shower. 

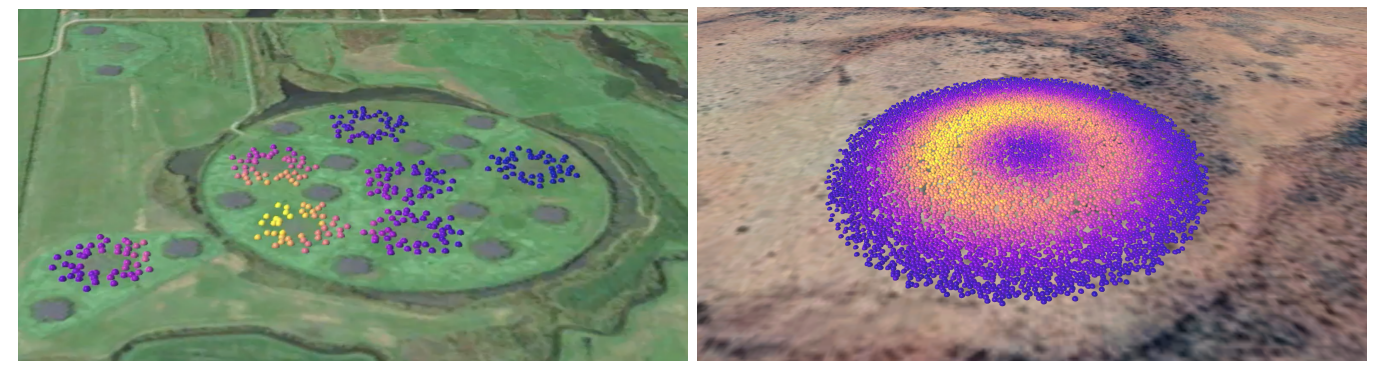

Figure 1: Left: Visualization of a typical air shower detection at LOFAR. The shower footprint is sampled by hundreds of antennas which are grouped in circular antennas stations. This data is fitted to radiation patterns simulated with CoREAS. Right: At the SKA, individual showers will be observed with thousands of antennas distributed much more homogeneously. The complete radiation pattern immediately becomes apparent.

\section{The SKA particle array}

The core of SKA-low is organized in 224 groups of 256 antennas. Between these groups, there is space to construct the SKA particle array. To achieve complete coverage of the core area with a spacing of roughly $100 \mathrm{~m}$, the array should consist of $80-100$ particle detectors. The exact number and spacing will depend on the final design of the antenna layout. Within these constraints the array configuration should be as homogeneous as possible.

The array is primarily used for triggering, and an initial reconstruction of the arrival direction and energy to check for consistency with the radio data. The final reconstruction of air showers will be purely based on the radio measurements. However, for composition studies, it will still be crucially important to have a precise understanding of the sensitivity of the detectors, in order to calculate trigger efficiencies.

A prototype detector has been deployed at the Murchison Radio-astronomy Observatory near the SKA-low site [12]. It consists of a scintillator plate of $\sim 1 \mathrm{~m}^{2}$ and four connected silicon photomultipliers. Communication is done through fiber optics cables to keep the production of radio interference below the limitations set by the observatory. The station has been operational for more than a year and runs stably. The performance is being studied to optimize the final design.

An improvement that is under consideration is to add muon-separation capability to the particle array by shielding some of the scintillators. This will further enhance the prospect of constraining hadronic physics in the air shower with the SKA [13].

\section{Ultra-high precision air shower observations}

To evaluate the performance of SKA as a cosmic-ray observatory, we did a simulation study following the same approach used at LOFAR [14]. That approach consists of the following steps.

For a shower with a specific arrival direction and energy, we run 30 full CORSIKA+CoREAS simulations. The random seeds were preselected with CONEX to ensure a good coverage of the entire naturally occuring range of $X_{\max }$. In these simulations, we use a star-shaped grid of reference antennas, which we use to generate two-dimensional maps of the intensity of the radio signal through 

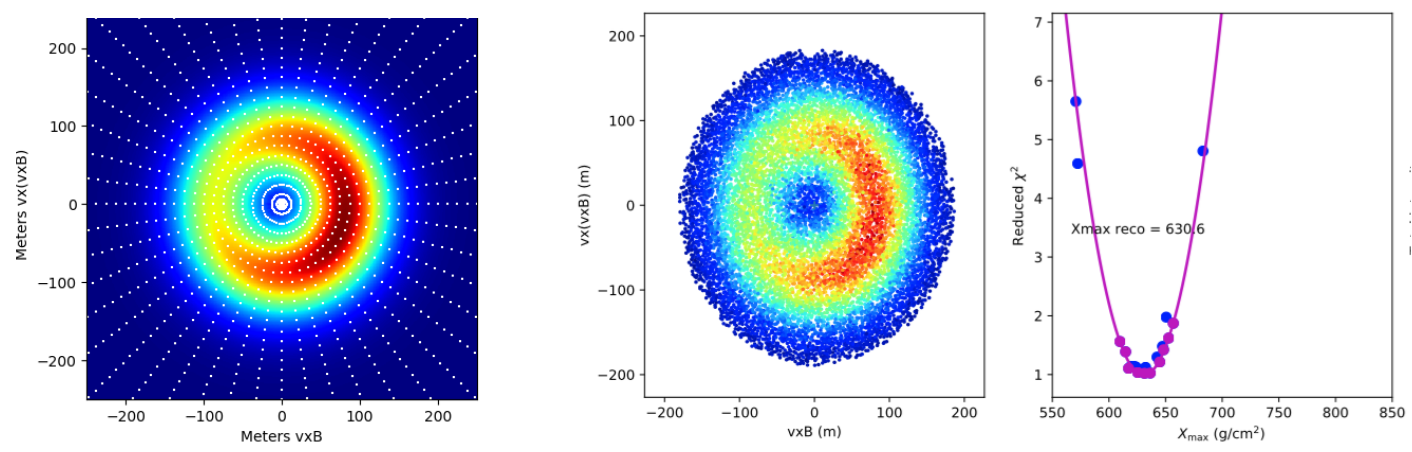

Figure 2: Left: simulation of radio intensity profile. The small white circles indicate the positions for which the full radio pulse was simulated with CoREAS. The complete picture is obtained through an interpolation method based on Fourier modes. Middle: this simulated event is observed with $\sim 8,500$ antennas simultaneously. Right: each simulated shower is fitted to the (mock) data. The reconstructed $X_{\max }$ is found by fitting a parabola through the data points in a plot of the reduced $\chi^{2}$ versus $X_{\max }$.

interpolation. All these maps are fitted to the data (either real data or a simulated shower with added noise) using as fit parameters the core position of the shower and a radio intensity scaling factor. In the final step, the reduced $\chi^{2}$ of the fits is plotted against $X_{\max }$. This yields a distribution with a clear minimum that can be fitted to obtain the reconstructed value for $X_{\max }$.

Several checks and improvements were needed to apply this technique to SKA conditions. For example, the use of interpolation to generate the two-dimensional shower profile is suspect. It can smooth out small-scale features to which the SKA will be very sensitive or introduce artifacts that degrade the performance. Furthermore, the difference in frequency range between SKA and LOFAR results in differently shaped radio profiles.

We simulated showers with very dense grids of reference antennas to quantify these effects. The details of this analysis will be provided in a forthcoming publication. The main conclusion is that an 8-arm star-shaped pattern with atenna spacing of $12.5 \mathrm{~m}$ (or larger at further distance from the core) can still be used, but a more accurate interpolation strategy is required which is based on Fourier modes ${ }^{1}$ instead of radial basis functions.

For the antenna layout of the SKA we used a randomly generated map of 60,000 antennas inside a circle of $500 \mathrm{~m}$ radius. This corresponds to the correct average antenna density and reflects the very homogeneous coverage of the actual design. We generated mock data by adding noise based on the galactic background radio emission and system noise (dominant above $\sim 200 \mathrm{MHz}$ ).

The simulation study yields a resolution of $6-8 \mathrm{~g} / \mathrm{cm}^{2}$ on $X_{\max }$ (compared to $20 \mathrm{~g} / \mathrm{cm}^{2}$ for LOFAR), an energy resolution of 3\% (LOFAR: 9\%) and a core resolution of $50 \mathrm{~cm}$ (LOFAR: 3 $-10 \mathrm{~m}$ ). While these values are already impressive, they are currently limited by the amount of simulated showers in the sample and can be improved further. In practice, the resolution will likely be determined by how well uncertainties of the current atmosphere and the antenna model can be constrained.

\footnotetext{
${ }^{1}$ We have made this code available via https://github.com/acorstanje/interpolation_fourier
} 


\section{Hadronic Physics}

The largest complication in determining the mass composition of cosmic rays is due to fundamental uncertainties in the hadronic interactions in the shower. Even with perfect knowledge of the $X_{\max }$ distribution, the inferred mass composition depends strongly on the adopted model [15].

Interestingly, different hadronic interaction models (e.g. QGSJETII.04, EPOS-LHC, and Sibyll2.3c) do not only predict different average $X_{\max }$ for specific nuclei, but also produce differently shaped longitudinal evolutions. These differences become most evident when expressing the longitudinal profile in terms of the $R$ and $L$ parameters, which describe the asymmetry and width of the profile respectively[16]. The Pierre Auger observatory has measured average values for $R$ and $L$ based on fluorescence measurements [17]. With a densely populated radio array, it is possible to reconstruct $L$ for individual showers [18].

The shape of the radio intensity profile on the ground is sensitive to $L$, but much less to $R$ as demonstrated in [18]. This can be understood heuristically by realizing the radio emission is coherent, so the radiated power is proportional to the square of the particle density. That means the total emission is not strongly affected by the edges of the longitudinal profile where the effect of asymmetry $(R)$ is strongest. The width of the profile, on the other hand, has direct consequences for the coherence conditions that apply to observers that see the shower under different angles.

The left panel in Fig. 3 illustrates how simultaneous reconstruction of $L$ and $X_{\max }$ can put strong constraints on hadronic physics in the shower (see caption for details). The question is if the effect of $L$ on the radio profile is strong enough to detect.

In fact, the sensitivity of the radio signal to $L$ has been hiding in plain sight for a while. The reduced $\chi^{2}$ curves that are used at LOFAR to reconstruct $X_{\max }$ are not perfect parabolas but have notable scatter. We have argued before that this could be due to 'shower-to-shower fluctuations other than $X_{\max }$ '. When the data points are colored to indicate the longitudinal width $L$, it becomes clear that $L$ is actually the dominant factor causing this scatter. This is shown in the right panel of Fig. 3.

While the effect of $L$ on the quality of fit is striking, it will be very hard to actually reconstruct $L$ with LOFAR. The reason is that the best $\chi^{2}$ value is not necessarily found for one particular value of $L$. Rather, it seems that a change in $L$ can be compensated with a shift in $X_{\max }$ leading to a similar quality of fit. This compensation requires some freedom in moving around the core position, which suggests that the SKA - with a core resolution of $50 \mathrm{~cm}$ - is the ideal instrument to reconstruct $L$. It is, however, not possible to draw definite conclusions with the limited amount of simulated showers available so far. A simultaneous fit of both $L$ and $X_{\max }$ requires a much larger set of reference showers.

A fast way to generate many showers is to use macroscopic simulation codes. MGMR3D [19] calculates radio pulses from an input longitudinal profile using a set of parametrizations that is based on CORSIKA simulations. While MGMR3D is many orders of magnitude faster than microscopic codes like CoREAS and ZHAireS, it is less detailed by design and it is not a priori clear whether it correctly predicts the influence of $L$.

We have studied the capabilties of MGMR3D by using it to reconstruct radio data generated with CoREAS. It was found that the values for $X_{\max }$ and $L$ reconstructed by MGMR3D have zenith-dependent offsets with respect to the true CORSIKA values. After introducing some simple 

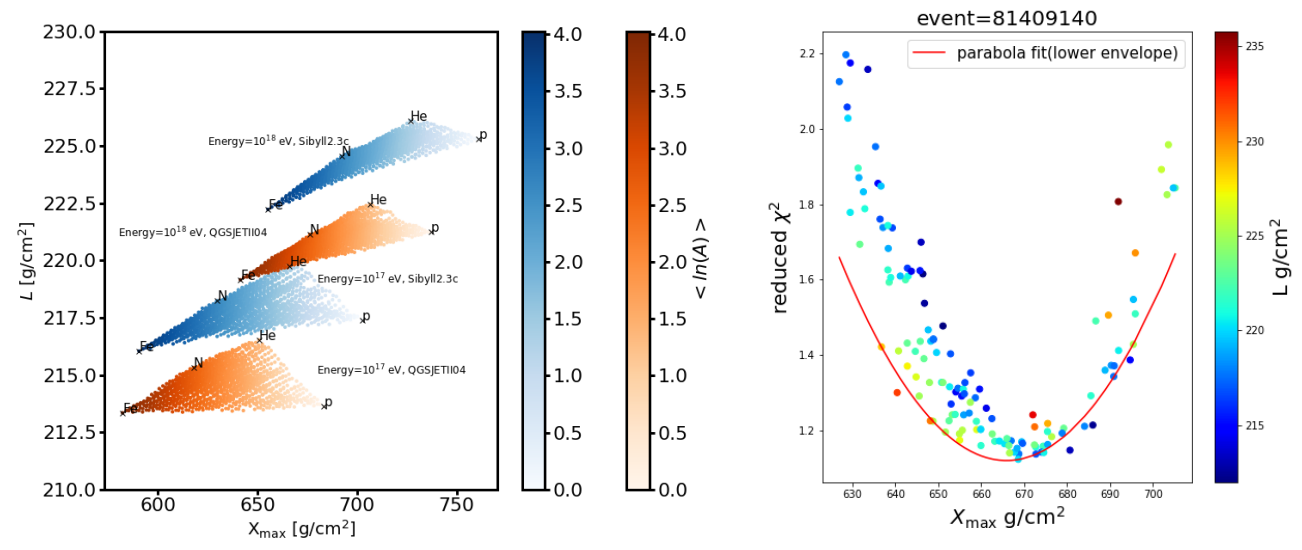

Figure 3: Left: each dot in this plot represents the average $X_{\max }$ and $L$ of a set of 5000 simulated shower using a specific mass composition consisting of proton, Helium, Nitrogen and Iron. For simulations of one particular energy and hadronic interaction model, a triangular shape is found when points are plotted for all different mixing ratios of elements. Some points on those triangles correspond to pure composition and are indicated in the plot. The four triangles correspond to two different energies and two hadronic interaction models. Even if the mass composition is completely unknown, QGSJETII-04 and Sibyll2.3c occupy different region in this plot, so recontructing $L$ will strongly constrain the models (Figure from [18]). EPOS-LHC has strong overlap with QGSJETII-04 and is omitted from this plot for clarity. Right: LOFAR data is fitted to simulations with different values for $X_{\max }$. The reconstructed $X_{\max }$ is found by fitting a parabola to the reduced $\chi^{2}$ values. By indicating the value of $L$ of each simulated shower with a color, it becomes clear that the fit quality strongly depends on $L$. This proves that $L$ can be reconstructed with a radio array of sufficient antenna density. This plot uses real LOFAR data.

scaling corrections, we achieve a resolution on $X_{\max }$ and $L$ of $\sim 10 \mathrm{~g} / \mathrm{cm}^{2}$ using a densely populated antenna grid. It is likely that this resolution will be further improved with microscopic simulations. A promising avenue is to use the template synthesis method that produces fast simulations while still maintaining the level of accuracy of a full CoREAS simulation [20].

Fig. 4 demonstrates the impact of reconstructing $L$ on astrophysical interpretation of the data. The plot contains a large variety of models, each a different combination of a Galactic component, extragalactic component, and hadronic interaction model. Details of the models can be found in [21]. In the $L-X_{\max }$ parameter space, the models occupy different areas. Without $L$ it is hardly possible to disentangle the contributions of astrophysics and the particle physics in the shower.

Note that Fig. 4 corresponds to a specific energy. Studying the evolution of these models with energy will provide additional constraints on the hadronic interaction models. Moreover, this plot is based on only the average values of $X_{\max }$ and $L$ while the shapes of their distribution will contain further information. For example $\sigma\left(X_{\max }\right)$ could be included in the analysis.

\section{Conclusion}

The extremely dense core of SKA will be a unique instrument for the observation of air showers. It can test radio simulation codes much more precisely than is currently possible, providing a 


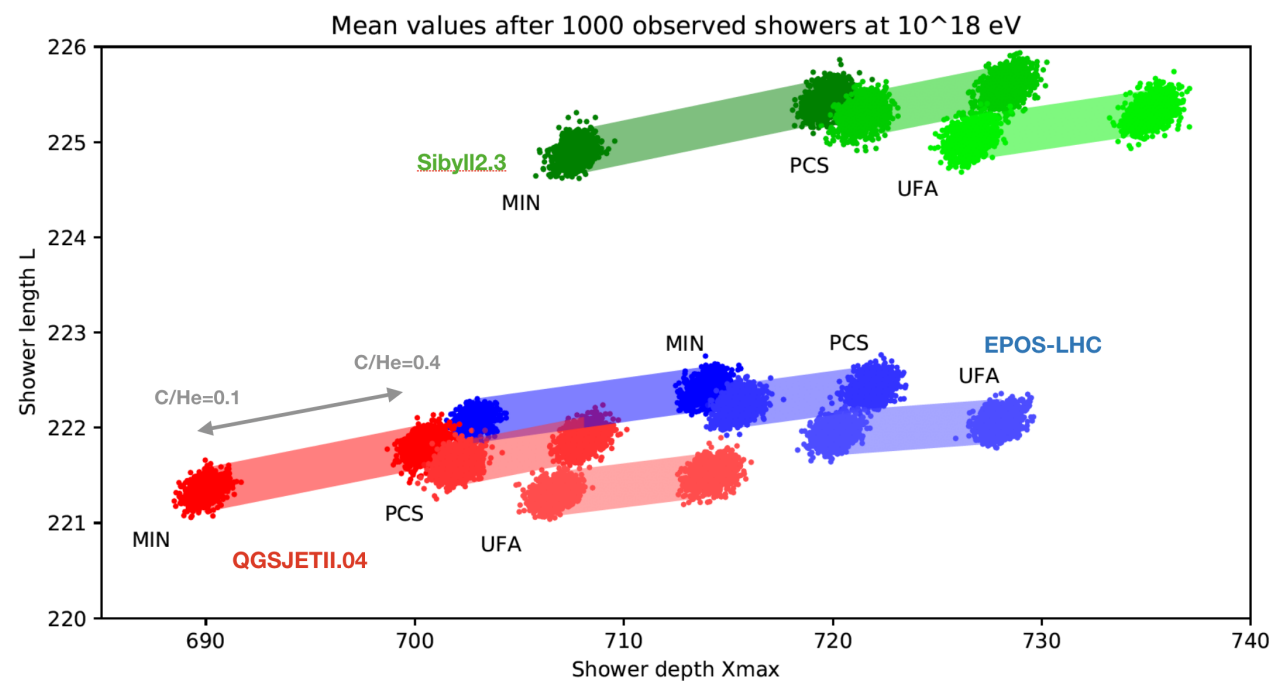

Figure 4: Simulation of average $X_{\max }$ and $L$ observations with SKA at $10^{18} \mathrm{eV}$. Each dot represents a possible outcome after observing 1000 showers including statistical uncertainties on $X_{\max }$ and $L$ of $10 \mathrm{~g} / \mathrm{cm}^{2}$. The different colors indicate hadronic interaction models. Each label indicates a different assumption for the extragalactic component (MIN, PCS, UFA). For the galactic component a prediction for Wolf-Rayet supernovas is used. Two extremes $(\mathrm{C} / \mathrm{He}=0.1$ and $\mathrm{C} / \mathrm{He}=0.4)$ were used in the calculation. Intermediate values are indicated with the colored bars connecting these two extremes. The grey arrow indicates which end of the bars corresponds to what element ratio. The different models occupy different regions in $L-X_{\max }$ space, but are highly degenerate in traditional measurements of only $X_{\max }$. Details of the models can be found in [21].

foundation for all future radio-based cosmic ray observations. It will be able to measure energy and $X_{\max }$ with unprecendented precision between $10^{16} \mathrm{eV}$ (or lower) $10^{18} \mathrm{eV}$. In addition, the shape of the longitudinal development of the shower can be reconstructed providing further constraints on source astrophysics as well as hadronic interactions in the shower. In order to achieve this potential, new reconstruction and simulation techniques need to be developed.

\section{References}

[1] HTTPS://WwW.SKATELESCOPE.ORG/WP-CONTENT/UPLOADS/2011/03/SKA-ASTOPHYSICSVoL1.PDF

[2] Huege, T. et al., "High-precision measurements of extensive air showers with the SKA", PoS ICRC 2015309 (2016), arXiv:1508.03465

[3] Hörandel, J., et al., "Precision measurements of cosmic rays up to the highest energies with a large radio array at the Pierre Auger Observatory", EPJ Web of Conf., 210 id.06005 (2019)

[4] Alvarez-Muñiz, J. et al., "The Giant Radio Array for Neutrino Detection (GRAND): Science and design”, Science China Phys., 63, Issue 1, article id. 219501, 43 pp. (2020). 
[5] Buitink, S. et al. [LOFAR], "A large light-mass component of cosmic rays at $10^{17}-10^{17.5}$ electronvolts from radio observations", Nature 531, 70 (2016).

[6] Corstanje, A. et al. [LOFAR], Depth of shower maximum and mass composition of cosmic rays from $50 \mathrm{PeV}$ to $2 \mathrm{EeV}$ measured with the LOFAR radio telescope, Phys. Rev. D 103, 102006 (2021).

[7] Zilles, A., Buitink, S., and Huege, T., "Extreme-precision measurements of cosmic rays via radio detection with the SKA", PoS ICRC 2017301 (2018), https://pos.sissa.it/301/360/

[8] Huege, T., Ludwig, M., and James, C.W., "Simulating radio emission from air showers with CoREAS”, AIP Conf. Proc.1535, Issue 1, p.128-132 (2013).

[9] Alvarez-Muñiz, J. et al., "Monte Carlo simulations of radio pulses in atmospheric showers using ZHAireS”, Astropart. Phys.35, 325 (2012).

[10] Schoorlemmer, H. and Carvalho Jr, W. "Radio interferometry applied to the observation of cosmic-ray induced extensive air showers" arXiv:2006.10348.

[11] Schlüter, F. and Huege, T., "Expected performance of air-shower measurements with the radio-interferometric technique", Journal of Instrumentation in press, arXiv:2102.13577

[12] Bray, J. et al., "The SKA particle array prototype: The first particle detector at the Murchison Radio-astronomy Observatory”, Nucl Inst. and Meth. in Phys. Res., A 973 , 164168 (2020).

[13] Schröder, F. et al., "Radio Detection of Cosmic Rays", Snowmass 2021 Letter of Interest (2021).

[14] Buitink, S. et al. [LOFAR], "Method for high precision reconstruction of air shower $\mathrm{X}_{\max }$ using two-dimensional radio intensity profiles", Phys. Rev. D 90, 082003 (2014).

[15] Pierog, T., "Hadronic interactions and air showers: where do we stand", ISVHECRI, EPJ Web of Conf. 208, 02002 (2018).

[16] Andringa, S., Conceição, R., Pimenta, M., "Mass composition and cross-section from the shape of cosmic ray shower longitudinal profiles", Astropart. Phys. 34 (20122) 360. (2011).

[17] Aab, A. et al. [Pierre Auger collaboration], "Measurement of the average shape of longitudinal profiles at the Pierre Auger Observatory", JCAP 03, 018 (2019).

[18] Mitra, P., "High Precision Reconstruction of Air Shower Properties With Dense Radio Arrays", $\mathrm{PhD}$ thesis at Vrije Univerisiteit Brussel (2021).

[19] Scholten, O. et al., "MGMR3D, a semi-analytic code for the obtaining the radio footprint from the shower currents", EPJ Web of Conf., 216, id.03003 (2019).

[20] Butler, D., "The Radio Signal of Cosmic Ray Air Showers and its Synthesis through Templates", $\mathrm{PhD}$ thesis at Karlsruhe Institute Technology (2020).

[21] Thoudam, S., et al., "Cosmic-ray energy spectrum and composition up to the ankle: the case for a second Galactic component", Astron. \& Astrophys., 595 A33 (2016) 